\title{
Direct Observation of Field-induced Modulation of Two-dimensional Electron Gas at Oxide Interfaces
}

Jinsol Seo ${ }^{1}$, Bumsu Park ${ }^{1,2}$, Christoph T. Koch ${ }^{3}$, Hyungwoo Lee ${ }^{4}$, Jungwoo Lee ${ }^{4}$, Chang-Beom Eom ${ }^{4}$ and Sang $\mathrm{Ho} \mathrm{Oh}^{1 *}$

1. Department of Energy Science, Sungkyunkwan University (SKKU), Suwon, Republic of Korea

2. Center for Nano Tubes and Nanostructured Composites, SKKU, Suwon, Republic of Korea

3. Department of Physics \& IRIS Adlershof, Humboldt University of Berlin, Berlin, Germany

4. Department of Materials Science and Engineering, University of Wisconsin-Madison, WI, USA

* Corresponding author: sanghooh@skku.edu

The recent discovery of a two-dimensional electron gas (2DEG) at the interface between insulating perovskite oxides $\mathrm{SrTiO}_{3}$ (STO) and $\mathrm{LaAlO}_{3}$ (LAO) was made possible by advances in atomic layercontrolled growth. It has been demonstrated that the $2 \mathrm{DEG}$ is localized to within a few nm of the interface [1], and that the carrier concentration can be altered with an electric field and/or lattice strain. Controlling such properties of the 2DEG by applying external biases has been demonstrated in various prototype device structures such as field effect transistors, diodes and nonvolatile memory devices. Eventually, seeing such changes in-situ during the operation of devices can improve our understanding and also provides a technical breakthrough for further in-depth characterization of such devices and ultimately optimization of their performance.

We have carried out in-situ TEM biasing experiments with the epitaxially grown LAO (10 u.c.)/STO (15 u.c.)/LAO (3 u.c.) devices to directly observe the modulation of the 2DEG by field effects. TEM samples for in-situ biasing experiment were prepared by using FIB (Fig. 1a). A thin lamella was lifted out and fixed on a Si MEMS chip and then further milled for electron transparency in FIB using a low energy $\mathrm{Ga}^{+}$ion beam. For the application of an electric field across the LAO/STO interfaces, an electrical circuit was constructed by cutting trenches for electrical isolation of one electrode from the other. While negative/positive $\mathrm{DC}$ biases are applied to the $\mathrm{SrRuO}_{3}$ top electrode, inline electron holography, STEM HAADF imaging and EELS have been carried out in-situ in a TEM/STEM system (GRAND ARM $300 \mathrm{CF}$ ). For all TEM samples $I-V$ curves were measured first to check the extent of leakage current and to select one that shows the lowest current for further elaborate in-situ experiments (Fig. 1b). The selected sample showed a $\sim \mathrm{nA}$ current level and a polarity-dependent asymmetric transport characteristic, which is related to the $180^{\circ}$ polarization switching of the $\mathrm{BaTiO}_{3}$ layer (Fig. 1a).

The charge density profiles obtained using inline electron holography [1] shows that the 2DEG at the lower LAO/STO interface (Interface II) responded to the applied voltage whereas that at the upper interface (Interface II) remained unchanged. More specifically, the 2DEG density at the lower interface increases when the negative bias increases exceeds $-0.8 \mathrm{~V}$, which can be attributed to an energy band bending effect. In-situ EELS measurements revealed a corresponding change of the Ti- $\mathrm{L}_{2,3}$ edge at this interface that is consistent with the change of the valence state of the $\mathrm{Ti}$ ion from +4 to +3 with accommodation of the extra 2DEG.

References:

[1] K. Song et al., Nature Nanotechnology 13 (2018) p. 198. 
[2] This work was supported by Samsung Research Funding \& Incubation Center of Samsung Electronics under Project Number SRFC-MA1702-01.

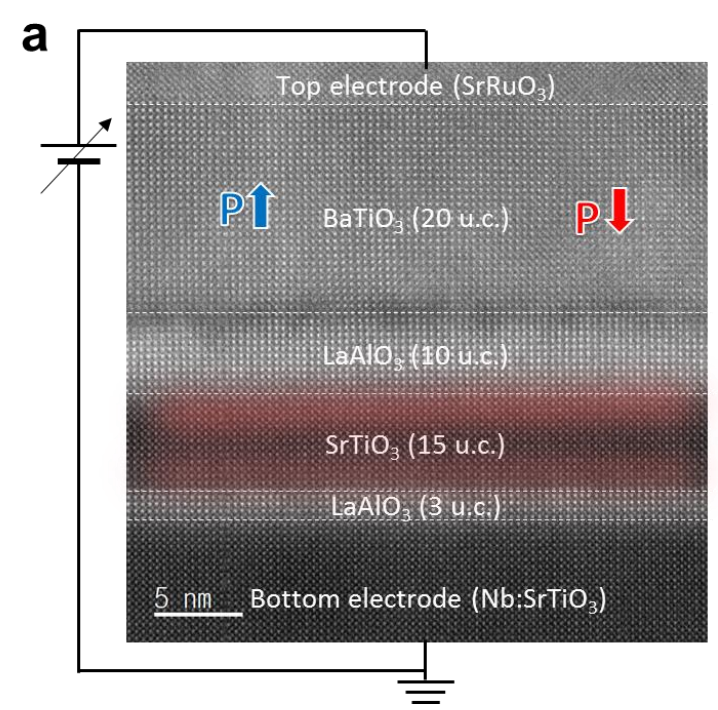

\section{b}

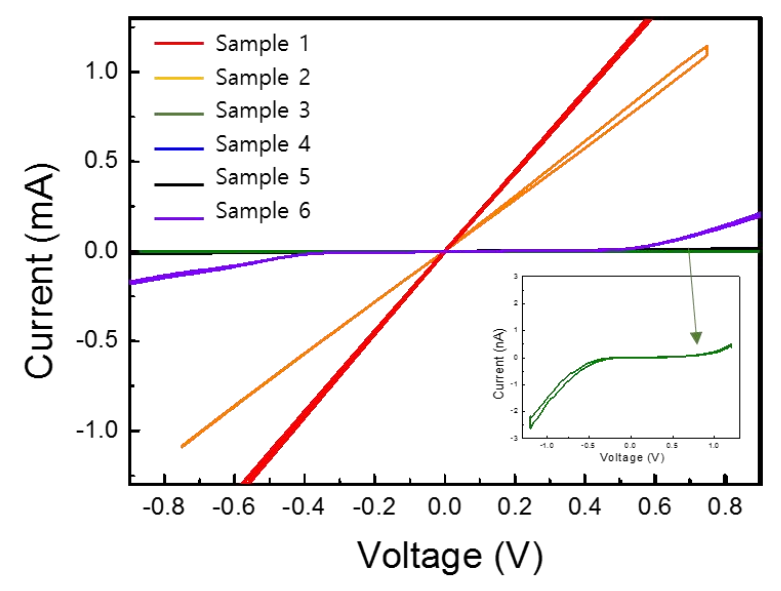

Figure 1. a, STEM HAADF image of the TEM sample prepared for in-situ biasing. The conductive LAO/STO interfaces with 2DEG are highlighted in red. b, I-V curves of different TEM samples. The inset I-V curve which exhibits the lowest leakage current level is considered as one that represents the intrinsic transport characteristic of this system.

\section{a}

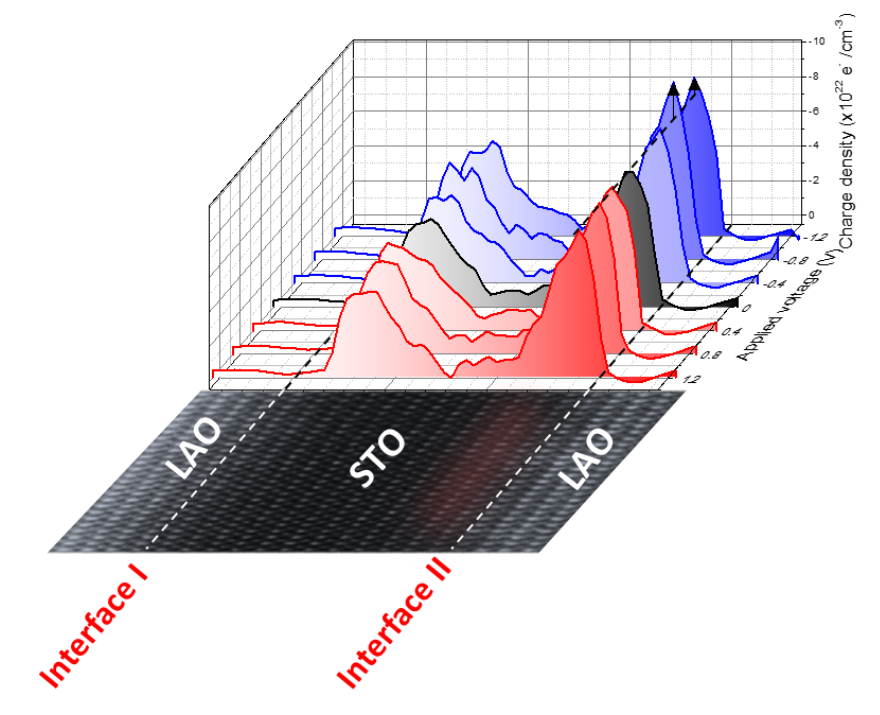

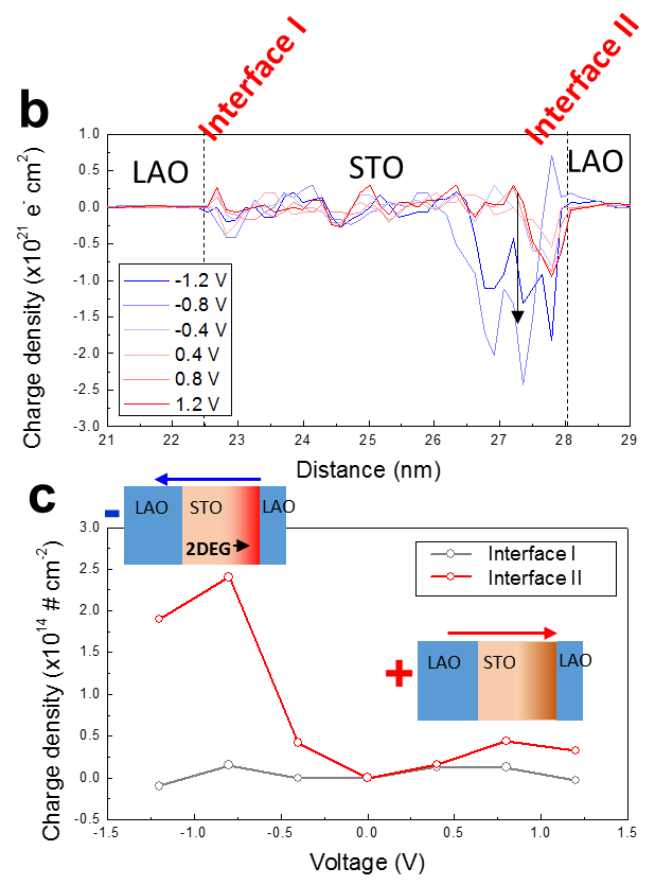

Figure 2. a, Charge density profiles across the LAO/STO/LAO interfaces, which were obtained by inline electron holography. b, Zero-bias subtracted charge density profiles showing the increase of charge density at the lower interface (Interface II) under negative biases. c, Change of 2DEG density at the upper interface (Interface I, gray) and at the lower interface (interface II, red) with applied biases. 